\title{
Dimensionality Reduction Techniques For Hyperspectral Image using Deep Learning
}

\author{
K.Sudha Rani, T.Gowri, T.Madhulatha
}

\begin{abstract}
This Research proposal addresses the issues of dimension reduction algorithms in Deep Learning(DL) for Hyperspectral Imaging (HSI) classification, to reduce the size of training dataset and for feature extraction ICA(Independent Component Analysis) are adopted. The proposed algorithm evaluated uses real HSI data set. It shows that ICA gives the most optimistic presentation it shrinks off the feature occupying a small portion of all pixels distinguished from the noisy bands based on non Gaussian assumption of independent sources. In turn, finding the independent components to address the challenge. A new approach DL based method is adopted, that has greater attention in the research field of HSI. DL based method is evaluated by a sequence prediction architecture that includes a recurrent neural network the LSTM architecture. It includes CNN layers for feature extraction of input datasets that have better accuracy with minimum computational cost.

Keywords: Hyperspectral Imaging(HSI), Dimensionality Reduction(DR), Deep Learning (DL), Independent Component Analysis(ICA),Principal Component Analysis(PCA),Minimum Noise Fraction(MNF).
\end{abstract}

\section{INTRODUCTION}

Human eye is able to distinguish between objects based on the respective spectral responses those responses are limited part of electromagnetic spectrum[1]. However, multispectral imaging sensors acquire in Fig.1,the electromagnetic spectrum bands. Its observed that the multispectral images uses only three bands among spectral range with huge band interludes, extends around visible region upto infrared regions of the spectrum.

images in simplest form whose bands are not necessarily contagious as seen in Fig.1 the electromagnetic spectrum bands. Its observed that the multispectral images uses only three bands among spectral range with huge band interludes, extends around visible region upto infrared regions of the spectrum. However due to this less number of spectral bands discrimination between various materials is the limiting

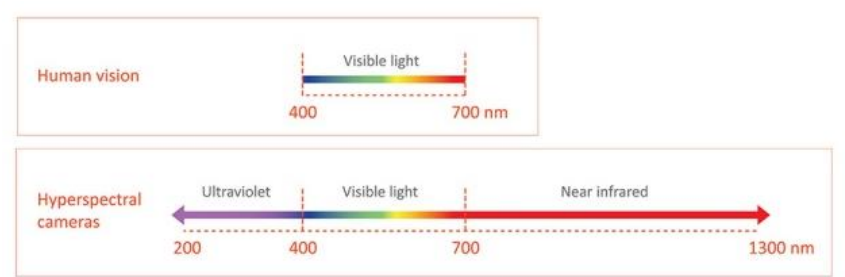

Fig.1:EM Spectral Bands

Revised Manuscript Received on December 13, 2019.

* Correspondence Author

TechnologyHyderabad,India. Email:kaligithisudha@gmail.com

T.Gowri, Senior Member,IEEE, ECE department, GITAM

University Vishakapatanam,India. Email:gowri3478@gmail.com.

T.Madhulatha, ECE department, MallaReddy Engineering College for

women,India. Email:madhulatha.t@gmail.com
K.Sudha Rani*, ECE department, TKR College of Engineering \&

factor[2]. With the involvement of hyperspectral sensing it is made possible to acquire several hundred bands with extremely intense frequency spectral resolution that can execute to discriminate subtle differences and ground covers[4] a clear illustration can be viewed in Fig.2.
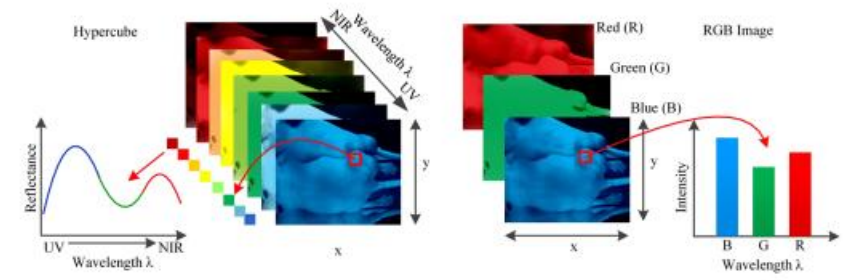

Fig.2: Multispectral Vs Hyperspectral Imaging

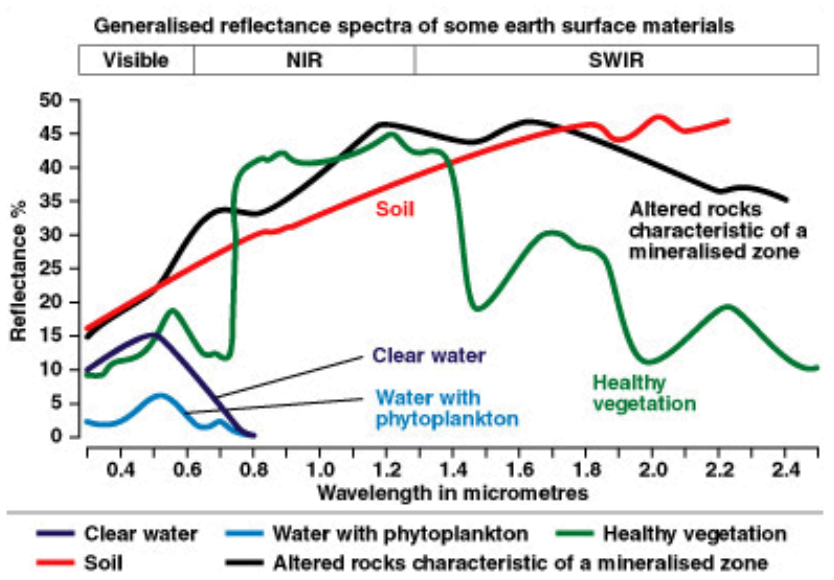

Fig.3: Spectral signatures of earth materials in different spectral regions

As hyperspectral imaging sensors acquires images in a very close spectral bands that results in a high dimensional data feature sets in spectral and spatial resolutions that contains redundant information[5].The increased spectral resolution of HSI allows in subtle discrimination of land services and different material by exploiting a property that each material has their respective spectral signatures, as seen in Fig..3, which was not possible previously due to low spectral resolution of multispectral imaging sensors[3].

\section{HYPERSPECTRAL IMAGING}

A remote sensing hyperspectral images (HSIs) been captured as a huge narrow, contiguous spectral wavelengths that ranges from $0.4 \mu \mathrm{m}$ to $2.5 \mu \mathrm{m}$ of visible light to infrared region of the electromagnetic spectrum that paves the way for wide range of applications of HSI in mining, agriculture, geology, and military surveillance. 


\section{Dimensionality Reduction Techniques For Hyperspectral Image using Deep Learning}

HSI can also be applied in numerous applications that includes the quality control of food and medical tests and examination, precision variability of agricultural lands , water resources and flood management, forensic examination of documents such as authentication of art works, handwritings, detection of military targets are certain systems that imply HSI with Deep Learning. HSI is categorized depending on the types of resolutions such as spatial and spectral.

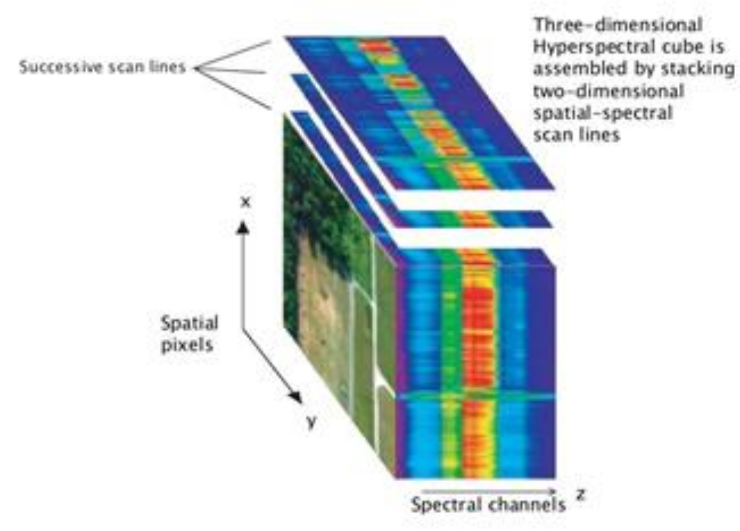

Fig. 4: Hyperspectral Image represented by a 3D cube The resolution can be defined as spatial if it is a measure of relatedness of pixels of an image with one another whereas the later resolution is a determination of variations with in pixels of an image to wavelength. HSI is a 3D cube that with two spatial data dimensions $\left(S_{x}, S_{y}\right)$ and one spectral data dimension $\left(S_{\lambda}\right)$. The spectral dimension results from the slices of the cube along the band or a channel as seen in Fig.4.

\section{A. SPATIAL RESOLUTION:}

To define resolution as spatial can be referred as the minute detectable feature of the image data which may lead to separate as similar part. Spatial resolution dictates the clarity of the image, these characteristics depends on imaging sensor. Spatial resolution and patch size are inversely proportional, which means as lesser the patch size the immense that details are elucidated.

\section{B. SPECTRAL RESOLUTION:}

This is defined as the number spectral bands and the extent of EM spectrum. For a low spectral resolution an HSI

imaging sensor acknowledges to large range of frequencies but may acquire defined number of interbands frequencies , however at high spectral resolutions sensor is sensitive to smaller range frequencies and captures massive number of frequency bands[3].Levels of spectral information includes identification ,detection, classification, quantification, and characterization. Few hyperspectral image datasets are listed in Table I.

Table-I : Spectral Sensors- Space and Airborne Datasets

\begin{tabular}{|c|c|c|c|c|}
\hline Sensor & $\begin{array}{c}\text { Organization } \\
\text { /Country }\end{array}$ & $\begin{array}{c}\text { Spect } \\
\text { ral } \\
\text { Band } \\
\mathbf{s}\end{array}$ & $\begin{array}{c}\text { Spectral } \\
\text { Range }(\boldsymbol{\mu m})\end{array}$ & $\begin{array}{c}\text { Spectral } \\
\text { Resolution }\end{array}$ \\
\hline Landsat-8 & NASA, US. & 8 & $0.45-12.50$ & 8 \\
\hline MODIS & NASA, US. & 36 & $0.40-14.40$ & $250-1000$ \\
\hline MERIS & ESA, EU. & 15 & $0.39-1.040$ & 300 \\
\hline Hyperion & NASA, US. & 242 & $0.40-2.500$ & 30 \\
\hline AVIRIS & NASA, US. & 224 & $0.38-2.500$ & $4-20$ \\
\hline
\end{tabular}

\begin{tabular}{|c|c|c|c|c|}
\hline HyMap & $\begin{array}{c}\text { Integrated } \\
\text { Spectronics Ltd, } \\
\text { Australia }\end{array}$ & 128 & $0.45-2.480$ & $2-10$ \\
\hline ROSIS & DLR, Germany & 115 & $0.42-0.873$ & 2 \\
\hline DAIS-7915 & GER Corp.,US & 79 & $0.45-12$ & $3-10$ \\
\hline AISA & SPECIM, Finland. & 286 & $0.45-0.9$ & 2.9 \\
\hline CASI & $\begin{array}{c}\text { Itres Research, } \\
\text { Canada. }\end{array}$ & 288 & $0.43-0.87$ & 2 \\
\hline
\end{tabular}

\section{C.SPECTRAL SIGNATURES AND RESPONSES OF HYPERSPECTRAL IMAGES:}

Hyperspectral Imaging Sensor acquires masses of contagious and exceedingly narrow bands that extends from mid to near infra-red regions of EM spectrum. Every pixel in HSI gives considerable information about the earth's surface material. Hyperspectral sensors measures all types of electromagnetic energy when it interacts with materials, it observes distinct features as per earth's materials.

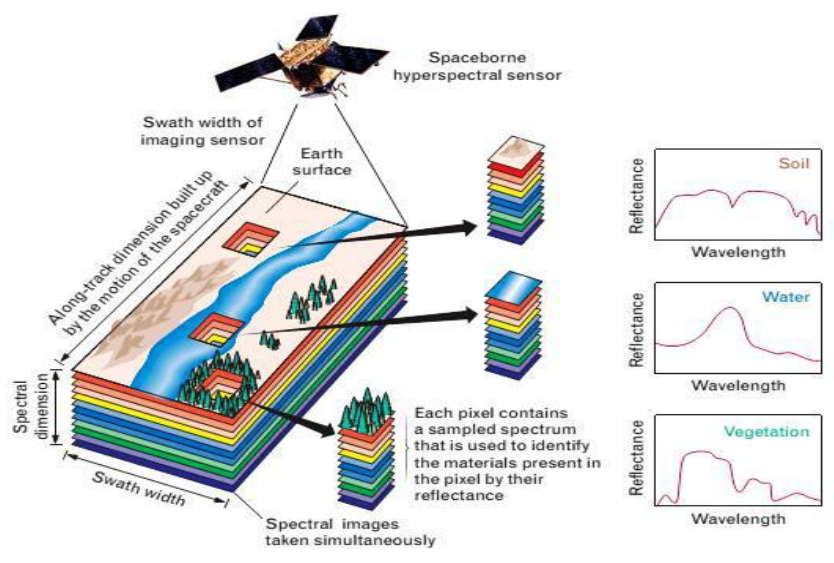

Fig 5: HSI mapping of each pixel to earths materials As viewed in Fig.5, Reflectivity can be stated as a measure of electromagnetic energy of every pixel that bounces off the earth's material surface, this is considered as the proportional change of reflected Vs incident energy with respect to the wave length, the reflectance values is different for different earth materials. The graphs are defined as spectral response curves to spectral signatures. However, the spectral resolution of the image sensor is proportional to classification information extracted from spectral signatures[2].

\section{DIMENSIONALITY REDUCTION}

HSI has various regions of spectra which a human eye can't detect. These number of bands in turn creates visible images from them which leads to an extremely detailed information about the scene, the classification of such enhanced spectral properties is a very formidable issue due to massive number of band values within every pixel that signifies the importance of dimensionality reduction.HSI image data sets has strong spectral correlation between the bands which implies that it has a considerable amount of redundant and misleading information[6] existing needs to be eliminated without any effective loss of information content. Reducing the high dimension data into lower dimension without sacrificing the significant information signifies as the prominent steps in processing of hyperspectral images. 
The data dimension is considered as the count of elements measured per observation. However, the limitation is it results large amounts of imaging data, though all the quantified measures are not significant for knowing the hidden phantasm[7].

The two main components that involve in reducing the dimensional features of an image are :

FEATURE SELECTION: considers a subset of the actual group of measured values as features that gives out a fewer subset, features that are used to model the problem. This happens in three ways that include Filter, Wrapper and Embedded[7]Feature Selection(FS) signifies the importance of classification accuracy.

FEATURE EXTRACTION: Method of retrieval of most important data, this targets extraction of the similar data defined as a label. Feature Extraction(FE) defines the related shape of the information defined as a pattern for ease of classification, which minimizes within class variability and enhances the variability between the classes[9]. The excessive the feature attributes, the sophisticated it becomes train the network.

\section{A.HSI FEATURE EXTRACTION TECHNIQUES FOR DIMENSIONALITY REDUCTION:}

The feature extraction methods of hyperspectral imaging can be categorized as shown in Fig.6, using Dimensionality reduction (DR) like linear and non linear methods that in turn includes supervised and unsupervised methods. Whereas, Multilayer methods for feature extraction [10] includes Deep learning like deep belief and convolution networks. Investigating the feature characteristics of hyperspectral image data finds an enormous ,contagious, narrow, multibands with strong band redundancy. This effects huge measures of data that consumes more amount of memories and speed of computation. Here finds the

purpose of dimensional reduction of hyperspectral images some techniques to reduce data dimensionality with effect to linear unsupervised leads to well known spectral feature extraction algorithms such as Principle Component Analysis (PCA) [11], Minimum Noise Fraction (MNF) [12], Independent Component Analysis (ICA) [13]. The virtues of dimensionality reduction is, it helps in data compression, in turn leads to reduced storage space. It also reduces computation time as well as removes redundant features. Dimensionality reduction seeks low-dimensional representation in lieu considering the entire bands [14],[15].DR algorithms under Spectral-analysis are categorized as supervised and unsupervised. The flow of exhibiting low dimensional data that includes no data label, can be referred as Unsupervised DR algorithms eg., PCA. The retention of data values within the same bounds referred as Supervised DR methods. However, the separation of data values using labeled samples among various classes is done using [16], [17],classifiers of Support Vector Machine (SVM).

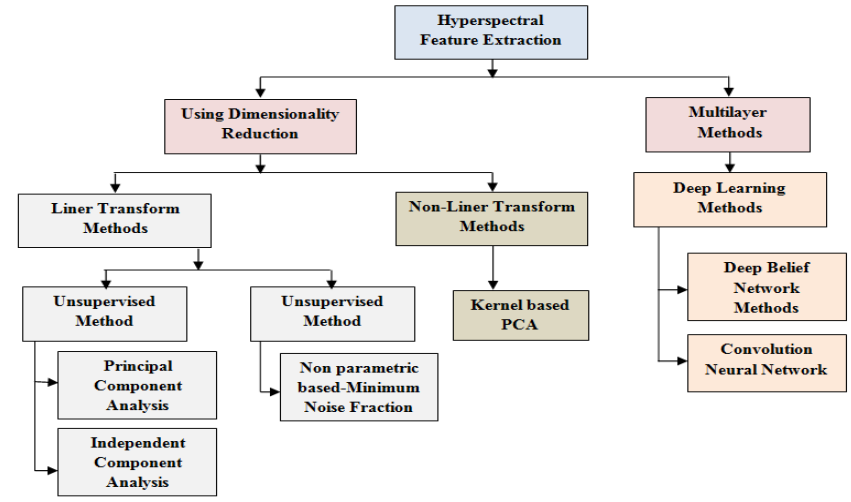

Fig.6: Hyperspectral Imaging Feature Extraction

Dimensionality reduction cuts down the time and storage space required and enhances the elucidation is viewed in Fig.7, that removes multi-co linearity between the data.

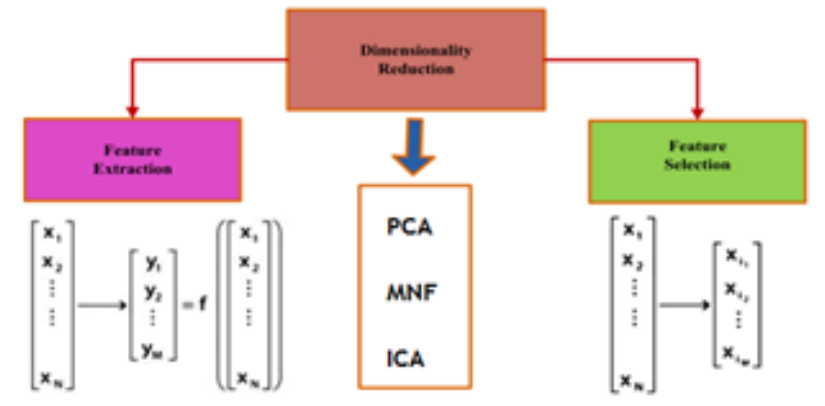

Fig.7: Dimensional Reduction

Also this brings down all high dimensional data to respective low dimensional which can be visualized as $2 \mathrm{D}$ or $3 \mathrm{D}$.

\section{PROPOSED APPROACHES}

\section{A. HSI Feature Extraction Using Dimensionality Reduction: PRINCIPAL COMPONENT ANALYSIS(PCA)}

PCA was introduced by Karl Pearson [18] that involves a matrix transformed to actual high dimensional data, is of the form of hyper ellipsoid that includes extensive range of correlation between frequency bands that renewed into other space that results in uncorrelated components ,these components are orthogonal with one and another. Quality of image is not considered its terms of SNR of PCA. Hence there is no specific order to term decrease in image quality will increase image component numbers to considerable range[18].However, this reduces subsequent processing steps leads to ease in reduction of the dimensions by PCA involves gauging datas of high measures to lower measures. In the lower dimensional space dataset variance should be maximum and computation time is reduced. PCA is held predominant for the reason of variable separation to the data content measure of a specific image as seen in Fig.8, shows a scatter plot along the axis $\mathrm{f} 1, \mathrm{f} 2$ with classification between two entities e1,e2 hence good class separation is not guaranteed in the transformed space. 


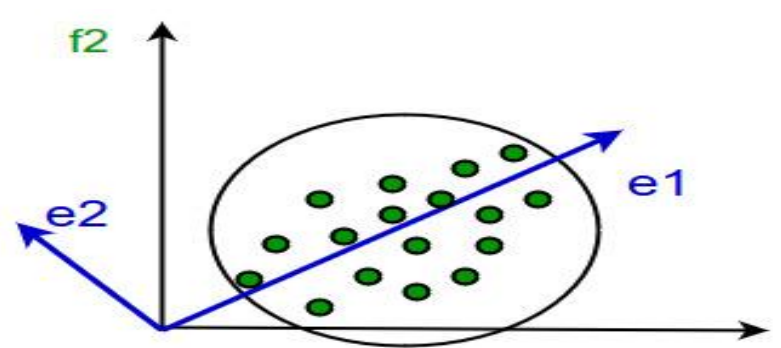

f1

Fig.8: Scatter Plot

The subjective investigation of the PC's is measured for the KSC AVIRIS scene results in the following subjective elucidation of data content within the bands related signifies the classes. The ordering of these PC's illustrates the problem such as,

\section{CLASSIFICATION OF HSI BASED ON PCA USING DEEP LEARNING:}

To evaluate the classification PCA-DBN method Deep belief network(DBN) is proposed followed by PCA, is a classic deep learning models works in defined order initially PCA reduces the HSI dimension later DBN is used for classification of PCA-DBN gives clearer edges, in comparison with other classification methods such as neural network(NN), support vector machine(SVM)[19] .

\section{CONTRAST OF PCA, KPCA AND ICA FOR DR:}

[20]This paper compares the performance of PCA, KPCA and ICA using feature extractions including SVM. These methods are used to transform higher-dimensional inputs into other lower-dimensional measure features. There are different types of features extraction methods .Specific algorithms adapted results in types of features extracted. The extracted features now becomes inputs of SVM to evaluate problems, resultant exhibits that PCA KPCA or ICA using Support Vector Machine supported by feature extraction is recommended over without feature extraction. However, of these methods the best performance is observed in KPCA that includes ICA feature extraction.

\section{MINIMUM NOISE FRACTION(MNF)}

Minimum noise fraction is a data transmission technique used to align the data along axes with respect to decrease in signal to noise (SNR) ratio.MNF maximizes the estimated SNR in hyperspectral imaging.

\section{SVM BASED CLASSIFICATION FOR HSI AFTER MNF ROTATION TRANSFORMATION:}

The MNF Rotation transforms drives the dimensionality reduction of data values, that exclude noise and also reduces the computational requirements[6]. It is a linear type of transformation which follows separate two level principal components analysis rotations, the first rotation uses PCA of noise matrix that is covariance to uncorrelate as well as to resize the noise present within the data. Next level includes the principal components obtained from the actual image data masked with white noise in first rotation and scale down by noise standard deviation. MNF a transform is a traditional DR procedure to acquire the essential feature bands. However the paper proposes that the component selection of MNF rotation transformation is analyzed with respect to SVM a classifier. The performance results with AVIRIS hyperspectral image dataset shows to keep around 1/10 MNF components [21].

INDEPENDENT COMPONENET ANALYSIS(ICA) ICA draws out source signals that are independent by distinguishing a linear and non-linear transformations that maximizes the analytical independence between various categories of classes present within the image [22].

The independent unmixing of signals is implemented using significant three major criteria's such as normality, complexity and independence are part of Blind Source separation. The hypothesis states that ICA are the source signals, they are mixed signal by nature and less complex they are statistically independent, their histograms are more shallow (less Gaussian) [23] [18].The signal components are reconstructed such that the latest attributes of the features are segregated, that are reflected from the first moment between the variables, whereas, in ICA components depends on higher order moments between the data measures which definitely is much stronger unmixing PCA.

On the other hand ICA reveals underlying hidden factors as it is a computational data sets of random variables, measurements or signals .A generative model for ICA [32]defines noticeable ranges of the data, that represents a huge amounts of samples. In this prototype, data variants are evaluated as linear combination of few investigated latent measures. The latent variables are defined as independent components which are nongaussian and mutually independent, these are referred as observed data. These independent components obtained stated as sources or factors.

\section{ICA BASED DIMENSIONALITY REDUCTION WITH APPLICATIONS IN HYPERSPECTRAL IMAGE ANALYSIS:}

[27]This paper examines ICA-DR generate all ICs and examines them to find which component to be retained. However for hyperspectral data that has hundreds of components will find limitation in selecting which components are really desired for the application. How to prioritize and select ICs plays a major role in finding the component[24][25]. Although eigen vectors have been used for this purpose it still has limitation of second order statistics as uses eigen analysis. This paper resolves the challenges by first using the concept to estimate the measure of spectrally distinct signatures that recommends to approximate measure of retained concepts dimensions referred as virtual dimensionality(VD) [26].

\section{B.HSI FEATURE \\ METHODS-MULTILAYER}

EXTRACTION

Hyperspectral data categorization follows a traditional model of pattern recognition, that happens by computing the raw input data features and later the obtained features are trained to work as classifiers, such as Support Vector Machines (SVM) and Neural Networks (NN) [27].

When a high dimensional datas are present the respective features are trained to perform significant learning methods to tackle the concept of high dimensionality and heterogeneity of hyperspectral data learning methods .

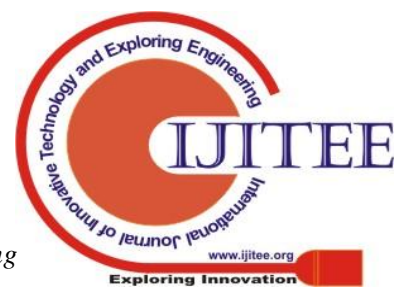


Various feature reducing methods are suggested including meager representation. $\mathrm{FS}$ is to observe a optimum subdivision of actual frequency spectral bands, while FE is to acquire a proper subgroup in a rebuilt feature space. In the dimension reduction the scale down features are used in place of the actual data to classify.

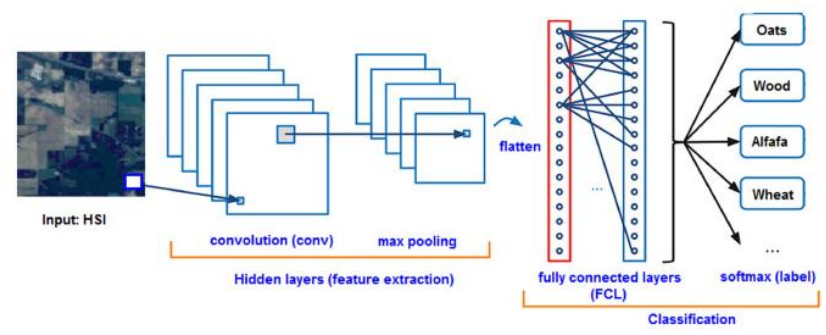

Fig. 9 CNN framework for Feature Extraction

Deep learning(DI ) is the latest arena after machine learnino that had achievı Fig. 9 CNN framework for Feature Extraction area of deep learning extends to indentity and diverse the spectral and spatio-spectral signatures of HSI. The high dense, narrow and contagious bands of hyperspectral images with respect to limited amount of labeled training data makes DL an appealing explorations in the fields of HSI

\section{DEEP SUPERVISED LEARNING FOR IMAGE CLASSIFICATION THROUGH CNN:}

The deep learning based approach for hyperspectral data classification exploits of CNNs and DLBs. The perspective is to construct hierarchically feature levels then translate pixels spectral and spatial information. Comparison over the methods to SVM-based classifiers, with widely hyperspectral datasets, represents the superior performance of deep learning approach with respect to image datasets. The interface of the training process, can be extended up to large datasets. The future prospective applications are extended upto framework development of unmasking human behavior through hyperspectral video sequences[28].The Convolution Neural Network framework is well directed by training specimens.Fig.9 represents the features realized at the outermost level of the convolution neural network ,stated flattened layer obtains single dimension feature vectors .

\section{HYPERSPECTRAL BASED IMAGE CLASSIFICATION USING DEEP RECURRENT NEURAL NETWORKS:}

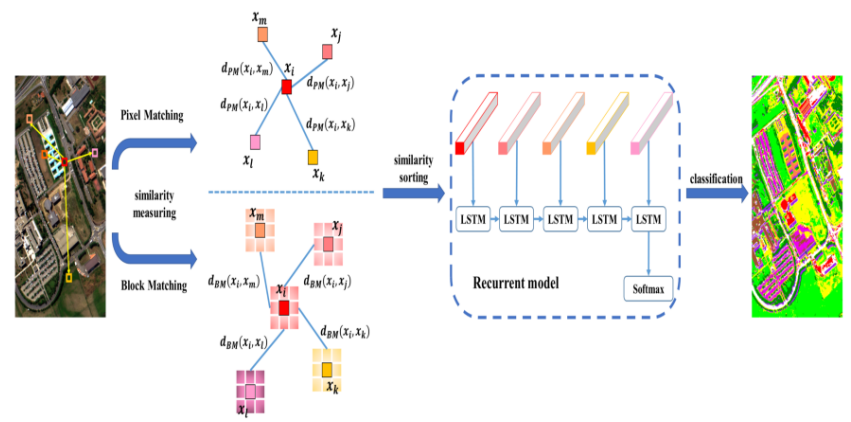

Fig.10:Classification using RNN-LSTM

Recurrent neural networks(RNN) referred as LSTM [29] were explored to extract temporal features from multiple data images. Although RNN based DL models significantly contributed to HSI processing. Limitations comes in the way of training a sample to acquire sufficient labeled training data for HSI as it is difficult and time consuming as DL based classification relies on the large sets of training samples.

LSTM HSI classification framework, where unlabeled data are well-exploited in order to construct sequential features from a single HSI. Instead of using spectral features as the sequential data structure of LSTM, pixels with similar data are gathered against the HSI data which are used to build the respective singular features. Upon construction, the similarity between a target pixel and all other pixels of the image are considered. To depict the similarity between two similarity-measuring strategies-pixel-matching and block-matching - are adopted here, Fig.10 shows where individual spectral features are used in pixel-matching-based schemes, where as both the methods are involved in block-matching-based schemes[30]

\section{RESULTS AND DISCUSSION}

The study include, two benchmark HSI datasets were utilized, including Pavia University, and Salinas images, as displayed in Figure 11 and Table II.

The Pavia University image data was collected by the Reflective Optics System Imaging Spectrometer (ROSIS) sensor. Image dataset consists of 102 frequency spectral bands, ranging from $430 \mathrm{~nm}$ to $860 \mathrm{~nm}$. The image has a spatial resolution of $1.3 \mathrm{~m}$, and the total image size is $610 \times 340$ pixels. For the Pavia University image extent, of nine (9) classes were considered in classification experiments. 


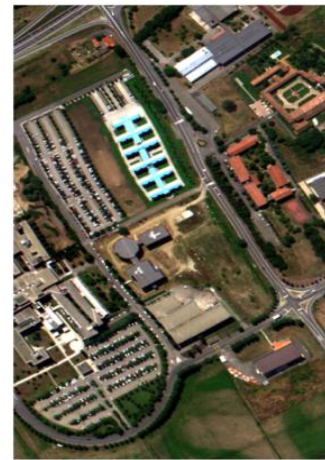

(a)

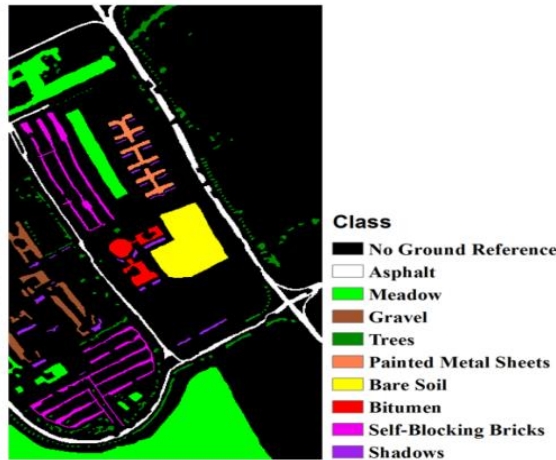

(b)

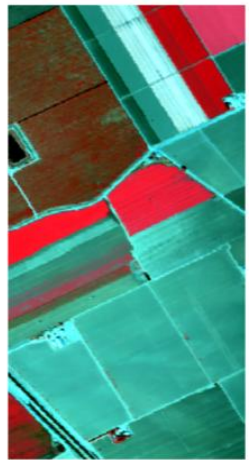

(c)

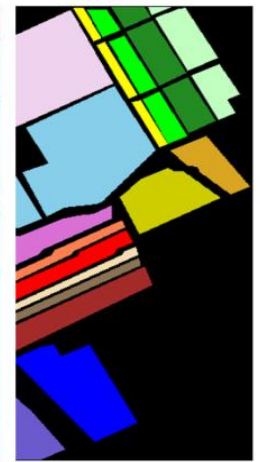

(d)

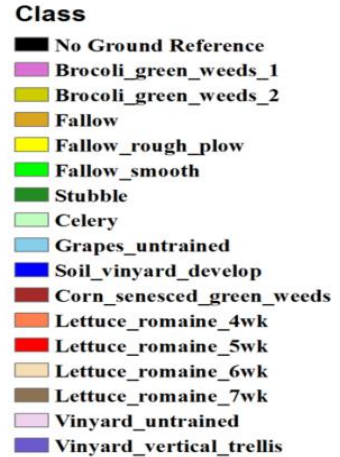

Vinyard_vertical_trellis

Fig.11: False-color image composites and their corresponding ground-reference data of Pavia and Salinas Images

The Salinas image was earned via the Airborne Visible/Infrared Imaging Spectrometer (AVIRIS), and the image contains 512 lines $\times 217$ samples, with the spatial resolution of $3.7 \mathrm{~m}$. .After removal of 20 noise and water-absorption bands, 204 spectral bands remained for subsequent analysis. the Salinas image entails 16 classes with respect to ground-reference data.

Table-II: Class Codes of Pavia and Salinas Images

\begin{tabular}{|c|c|c|c|}
\hline \multicolumn{2}{|c|}{ Pavia University Image } & \multicolumn{2}{|r|}{ Salinas Image } \\
\hline $\begin{array}{l}\text { Class } \\
\text { No. }\end{array}$ & Name & $\begin{array}{l}\text { Class } \\
\text { No. }\end{array}$ & Name \\
\hline 1 & Asphalt & 1 & Brocoli_green_weeds_1 \\
\hline 2 & Meadow & 2 & Brocoli_green_weeds_2 \\
\hline 3 & Gravel & 3 & Fallow \\
\hline 4 & Trees & 4 & Fallow_rough_plough \\
\hline 5 & $\begin{array}{l}\text { Painted Metal } \\
\text { Sheets }\end{array}$ & 5 & Fallow_smooth \\
\hline 6 & Bare Soil & 6 & Stubble \\
\hline 7 & Bitumen & 7 & Celery \\
\hline 8 & $\begin{array}{c}\text { Self-Blocking } \\
\text { Bricks }\end{array}$ & 8 & Grapes_untrained \\
\hline 9 & Shadows & 9 & Soil_vineyard_develop \\
\hline & & 10 & Corn_sensced_green_weeds \\
\hline & & 11 & Lettuce_romaine_4wk \\
\hline & & 12 & Lettuce_romaine_5wk \\
\hline & & 13 & Lettuce_romaine_6wk \\
\hline & & 14 & Lettuce_romaine_7wk \\
\hline & & 15 & Vinyard_untrained \\
\hline & & 16 & Vinyard_vertical_trellis \\
\hline
\end{tabular}

DISCUSSION

\section{CONCLUSION}

The Dimensionality Reduction of Hyperspectral Imaging using Deep Learning is an ICA-stationed feature extraction method that extracts independent components later detects targets for various data sets of hyperspectral imaging sensors. Fig.12, indicates the procedure of preprocessing of acquired input followed by feature extraction using ICA the dimensionality reduction procedure preprocessing of for feature extraction later the Independent Component Analysis (ICA) is used to classify the hyperspectral image sets into labels depending on the spectral signatures which depend on training samples upon exploring and matching with their respective spectral signatures only gives out ground truth values of surface materials.

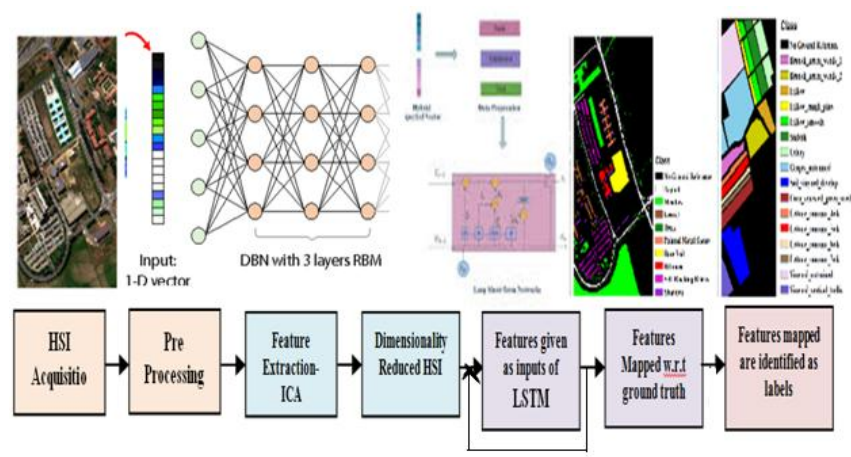

\section{REFERENCES}

Fig.12: Proposed Architecture

1. E. H. Land and J. McCann, "Lightness and retinex theory," JOSA, vol. 61, no. 1, pp. 1-11, 1971.

2. Muhammad Jaleed Khan, Hamid Saeed Khan, Adeel Yousaf, Khurram Khurshid, Asad Abbas, " Modern Trends in Hyperspectral Image Analysis: A Review", IEEE , Vol.6, pp.14118-14126, 2018.

3. D. Landgrebe, "Hyperspectral image data analysis," IEEE Signal Processing Magazine, vol. 19, no. 1, pp. 17-28, 2002

4. Plaza A, Martinez P, Plaza J, Perez RM. Dimensionality reduction and classification of hyperspectral image data using sequences of extended morphological transformations. IEEE T Geosci Remote 2005; 43 466-479.

5. Junying $\mathrm{S}$, Ning $\mathrm{S}$. A dimensionality reduction algorithm of hyper spectral image based on fract analysis. In: 21st ISPRS Congress Technical Commission VII, International Archives of the Photogrammetry, Remote Sensing and Spatial Information Sciences; 3-11 July 2008; Beijing, China. pp. 297-302.

6. "Dimensionality Reduction Techniques for Hyperspectral Images" Shraddha P. Lodha1 and Prof. S. M. Kamlapur2, International Journal of Application or Innovation in Engineering \& Management (IJAIEM) WebSite: www.ijaiem.org Email: editor@ijaiem.org Volume 3, Issue 10, October 2014 ISSN 2319 - 4847

7. Jihan Khodr, Rafic Younes"Dimensionality reduction on hyperspectral images: A comparative review based on artificial datas"2011 IEEE

8. D Lakshmi Padmaja Dr.B Vishnuvardhan "Comparative Study of Feature Subset Selection Methods for Dimensionality Reduction on Scientific Data" 2016 IEEE.

9. Gaurav Kumar,Pradeep Kumar Bhatia"A Detailed Review of Feature

10. Extraction in Image Processing System"2014 IEEE

11. Radhesyam Vaddi, Dr M Prabukumar "Comparative Study of Feature Extraction Techniques For Hyper Spectral Remote Sensing Image Classification : A survey"IEEE 2017 
12. P. Bajorski. "Statistical inference in pca for hyperspectral images". IEEE J. Sel. Topics Signal Process., 5(3):438-445, June 2011.

13. Asgeir Bjorgan,Lise Lyngsnes Randeberg, "Real-Time Noise Removal for Line-Scanning Hyperspectral Devices Using a Minimum Noise Fraction-Based Approach". Sensors 2015, 15, 3362-3378. OPEN ACCESS sensors ISSN 1424-8220 www.mdpi.com/journal/sensors.

14. A. Villa, J. A. Benediktsson, J. Chanussot, and C Jutten."Hyperspectral image classification with independen component discriminant analysis". IEEE Trans. Geosci. RemoteSens., 49(12):4865-4876, Dec. 2011

15. C. Lee and D. A. Landgrebe, "Analyzing high-dimensional multispectral data," IEEE Trans. Geosci. Remote Sens., vol. 31, no. 4, pp. 792-800, Jul. 1993

16. W. Li, S. Prasad, J. E. Fowler, and L. M. Bruce, "Locality-preserving dimensionality reduction and classification for hyperspectral image analysis," IEEE Trans. Geosci. Remote Sens., vol. 50, no. 4, pp. 1185-1198, Apr. 2012

17. S. Chen and D. Zhang, "Semisupervised dimensionality reduction with pairwise constraints for hyperspectral image classification," IEEE Geosci. Remote Sens. Lett., vol. 8, no. 2, pp. 369-373, Mar. 2011

18. Q. Shi, L. Zhang, and B. Du, "Semisupervised discriminative locally enhanced alignment for hyperspectral image classification," IEEE Trans. Geosci. Remote Sens., vol. 51, no. 9, pp. 4800-4815, Sep. 2013

19. Jolliffe.I "Principal Component Analysis"(2ed., Springer,2002) (518s)_MVSa.pdf

20. Kitti Koonsanit, Chuleerat Jaruskulchai, and Apisit Eiumnoh,

21. "Band Selection for Dimension Reduction in Hyper Spectral Image Using Integrated Information Gain and Principal Components Analysis Technique "on International Journal of Machine Learning and Computing, Vol. 2, No. 3, June 2012

22. Qiaoqiao Sun ; Xuefeng Liu ; Min Fu"Classification of hyperspectra image based on principal component analysis and deep learning",IEEE 2017.

23. " PCA, Kernel PCA and Dimensionality Reduction in Hyperspectral Images",Aloke Datta, Susmita Ghosh and Ashish Ghosh (C) Springe Nature Singapore Pte Ltd. 2018 G. R. Naik (ed.), Advances in Principal Component Analysis.

24. Zhang Denghui; Yu Le" Support Vector Machine Based Classification for Hyperspectral Remote Sensing Images after Minimum Noise Fraction Rotation Transformation",IEEE 2011

25. Onlinesource:"https://en.wikipedia.org/w/index.php?title=Hyperspect ral_imaging\&oldid=887669877" on 14 March 2019

26. M. P. Uddin , M. A. Mamun and M. A. Hossain," Improved Feature Extraction Using Segmented FPCA for Hyperspectral Image Classification", 2nd International Conference (ICEEE) December 2017

27. C.-I Chang, Hyperspectral Imaging: Techniques for Spectral Detection and Classification. Norwell, MA: Kluwer, 2003.

28. C.-I Chang and Q. Du, "Estimation of number of spectrally distinct signal sources in hyperspectral imagery," IEEE Trans. Geosci. Remote Sens., vol. 42, no. 3, pp. 608-619, Mar. 2004

29. Jing Wang, Student Member, IEEE, and Chein-I Chang, Senior Member," Independent Component Analysis-Based Dimensionality Reduction With Applications in Hyperspectral Image Analysis", IEEE Transactions On Geoscience And Remote Sensing, Vol. 44, No. 6 , June 2006

30. G. Camps-Valls and L. Bruzzone, "Kernel Methods for Remote Sensing Data Analysis", J. Wiley and Sons, NJ, USA, 2009.

31. Konstantinos Makantasis , Konstantinos Karantzalos , Anastasios Doulamis , Nikolaos Doulamis"Deep Supervised Learning For Hyperspectral Data Classification Through Convolutional Neural Networks" IEEE 2015

32. Hochreiter, S.; Schmidhuber, J.," Long short-term memory. Neura Comput." 1997, 9, 1735-1780. [CrossRef]

33. Andong Ma, Anthony M. Filippi, Zhangyang Wang and Zhengcong Yin "Hyperspectral Image Classification Using Similarity Measurements-Based Deep Recurrent Neural Networks "

34. A. Villa, J. A. Benediktsson, J. Chanussot, and C. Jutten Hyperspectral image classification with independent componen discriminant analysis. IEEE Trans. Geosci. Remote Sens., 49(12):4865-4876, Dec. 2011.

\section{AUTHORS PROFILE}

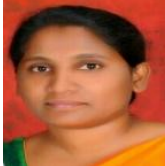

First Author: K. SUDHA RANI presently working as Associate professor in the department of Electronics and Communication Engineering at TKR College of Engineering \& Technology, Medbowli, Meerpet, SaroorNagar, Hyderabad, Telangana State, INDIA. She has 12 years of teaching experience. She has obtained B. Tech. degree in Electronics and Communication Engineering from Jyothishmath Institute of Technological Sciences, Karimnagar, Jawaharlal Nehru Technological University Hyderabad, in 2005, M.Tech. degree in Embedded Systems from CMR College Of Engineering \&Technology,Hyderabad,Jawaharlal Nehru Technological University Hyderabad, in 2010 and my area of Research interest is Computer Vision \& Image Processing, Ph.D (ECE) from GITAM University, Vishakapatnam and it is my part of Research work.

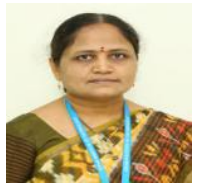

Second Author: Gowri Thumbur received the B.Tech degree in electronics and communication engineering from VR Siddartha college, Vijayawada, under Nagarjuna University, Guntur, India, in 2000 ande M.Tech. degree in electronics and communication engineering from Jawaharlal Nehru Technological University Anantapur, Anantapur, India, in 2005, and the Ph.D. degree in electronics and communication engineering (signal processing) from Jawaharlal Nehru Technological University Kakinada, Kakinada, India. She is currently with the Department of Electronics and Communication Engineering, GITAM Institute of Technology, GITAM University, Visakhapatnam, India. She has several publications in reputed journals. Her research interests include signal processing, VLSI, digital image processing, and information security. Dr. Thumbur Gowri is a life member in ISSS and senior IEEE member.

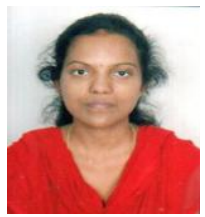

Third Author:T.Madhulatha presently working as Assistant Professor in the department of ECE at MallaReddy Engineering College for women, Maisammaguda, dhulapally, secunderabad, Telangana, India. She has 9years of teaching experience. She has obtained B.Tech degree in Electronics and

\section{Communicatio}

engineering from KSRM college of Engineering, Kadapa, Sri Venkateswara University, Tirupati in 2007, M.Tech degree in Digital Electronics and Communication System (DECS) from KSRM college of engineering, kadapa , JNTUA, Anantapur in 2013 and my area of research interest in Digital Image Processing, $\mathrm{PhD}$ (ECE) from GITAM University, Visakhapatnam and it is my part of research work. 